\title{
Standards make the world go round
}

\author{
Domenica D’Elia ${ }^{1 凶}$, Chris Evelo ${ }^{2,3}$, Babette Regierer ${ }^{4,5}$, Susanne Hollmann ${ }^{4,6}$ \\ 'Institute for Biomedical Technologies, National Research Council (CNR), Bari, Italy \\ ${ }^{2}$ Department of Bioinformatics - BiGCaT, Maastricht University, Maastricht, Netherlands \\ ${ }^{3}$ Maastricht Centre for Systems Biology (MaCSBio), Maastricht University, Maastricht, Netherlands \\ ${ }^{4}$ SB Science Management UG, Berlin, Germany \\ ${ }^{5}$ Leibniz Institute for Vegetable and Ornamental Crops (IGZ), Großbeeren, Germany \\ ${ }^{6}$ Potsdam University, Potsdam, Germany \\ Competing interests: DD none; CE none; BR none; SH none
}

On the 2 March 2020, COST Action - Harmonising standardisation strategies to increase efficiency and competitiveness of European life-science research $\left(\mathrm{CHARME}^{1}\right)$ - held its final conference in Brussels.

After four years of successful work, the members of the COST Action CHARME met in Brussels to summarise the achievements and to discuss future perspectives and challenges for standardisation in the life sciences.

Standards represent important drivers in the lifesciences and technology transfer because they guarantee that data become accessible, shareable and comparable along the value chain.

The CHARME network, chaired by Dr Susanne Hollmann, fostered collaboration between researchers from 31 countries to increase awareness for the need for standards, enabling the reuse of research data and their interoperability within the community. CHARME provides a common ground for researchers from academia, research institutes, SMEs and multinational organisations.

Following the motto "Standards make the world go round", the outcomes of the COST Action are manifold and introduced some basic concepts and definitions that support a better understanding of the challenges and requirements.

A challenge identified is the digitalisation and interoperability of data and tools in wet- and in silicolabs, because there is an urgent need for common languages and ontologies to enable data reuse and process automation. This becomes an even bigger challenge if researchers work with data derived from different scientific fields. Relevant instruments to support the implementation of standardisation are tools for data and process documentation. Unfortunately,

${ }^{1}$ https://www.cost.eu/actions/CA15110/\#tabs|Name:overview the interoperability between the existing instruments is limited. Hence, the development of new tools is necessary to allow the transfer of data from one system to another and thus allowing the reuse of data from databases and data repositories. Despite the fact that many researchers already make their data compliant to the FAIR principles (Findable, Accessible, Interoperable and Reusable), identifiers or terms used are not harmonised (e.g. database identifiers, ontologies and chemical [sub] structures).

The extensive involvement of the scientific community in CHARME is one of the most important achievements of this COST Action. CHARME participants have attracted, interacted and cooperated intensively with international organisations such as the ISO TC $276^{2}, \mathrm{CEN} / \mathrm{CENELEC}^{3}$, with initiatives and scientific organisations like ELIXIR ${ }^{4}$, ORPHANET ${ }^{5}$, COMBINE $^{6}$, FAIRDOM ${ }^{7}$, GA4GH $^{8}$.

To harmonise activities with the efforts done outside Europe, CHARME was supported by the COST Association $^{9}$ to run a joint workshop ${ }^{10}$ with the Massive Analysis and QC (MAQC $\left.{ }^{11}\right)$ Society from the US where both communities discussed fundamental themes of research and machine learning reproducibility in the context of standard's needs.

During European and international conferences and workshops ${ }^{12}$, CHARME has presented possible solutions in the development and implementation of a uniform

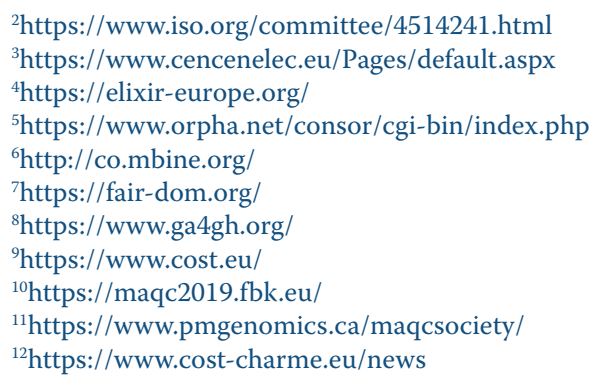

\section{Article history}

Received: 20 March 2020

Published: 26 March 2020

(c) 2020 D'Elia et al.; the authors have retained copyright and granted the Journal right of first publication; the work has been simultaneously released under a Creative Commons Attribution Licence, which allows others to share the work, while acknowledging the original authorship and initial publication in this Journal. The full licence notice is available at http://journal.embnet.org. 


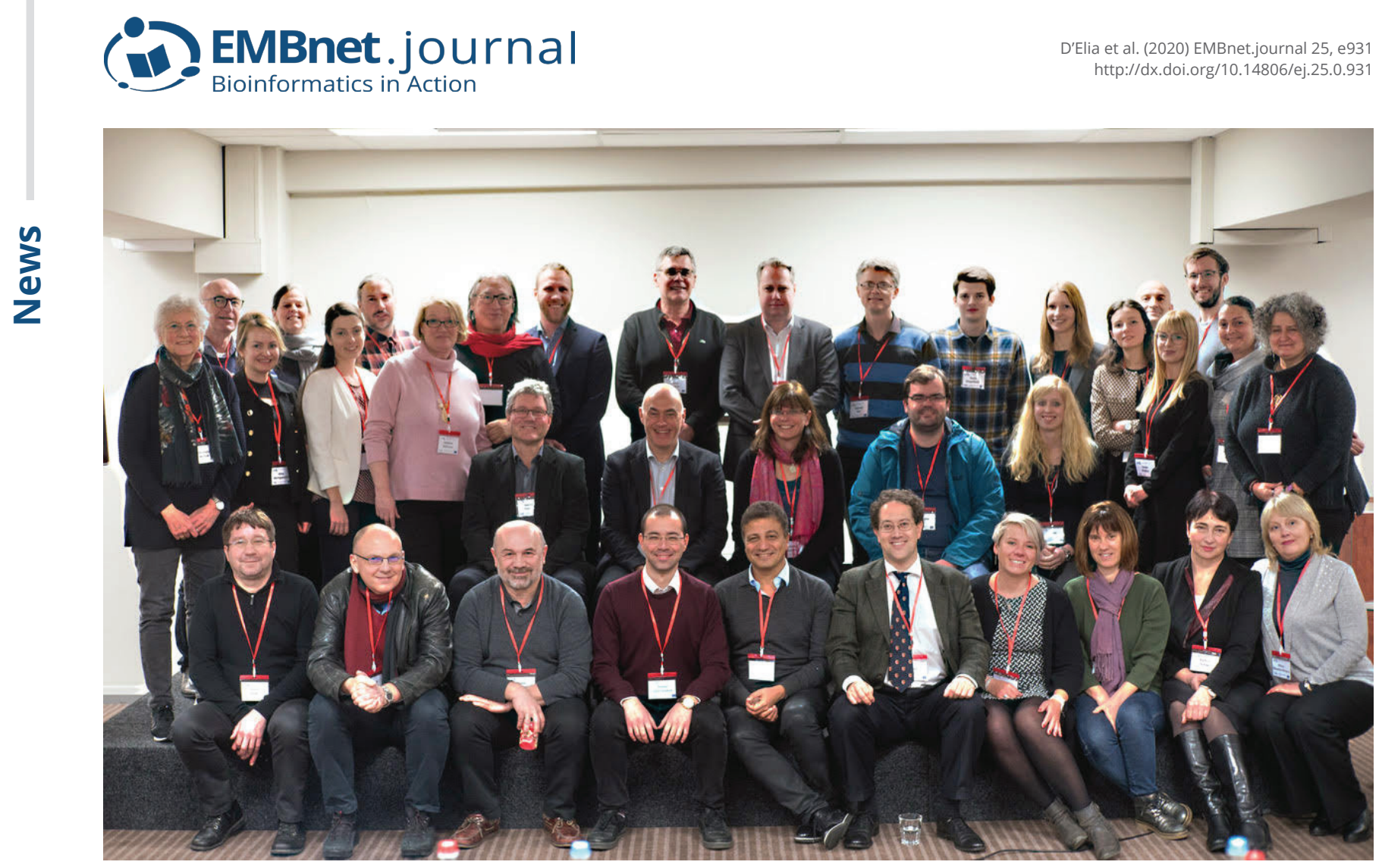

Figure 1. Group picture of attendants at the CHARME Final Conference, 2-3 March 2020, Brussels.

European Education \& Training programme in existing curricula.

An excellent training programme was realised through the organisation of Think Tank events, training schools and an effective set of STSM actions. Young researchers participating in the first training school, organised in 2017, were so much motivated by the standardisation topic that they initiated followup schools and activities. Noteworthy, 15 Early Stage Researchers coming from 12 member countries and hosted in another 7 member countries benefited from STSMs. Furthermore, as part of this success story, the outcome of these STSMs inspired new collaborations, master theses, new project ideas and are also reflected in publications. All beneficiaries of the STSM networking tool agreed that the STSM has been of high value for both training and career.

The results of this COST Action's network will be subject of a White Paper addressing the needs of standardisation, including a catalogue of requirements and recommendations to be disseminated to decisionmakers at all levels to enable the implementation of standards in the daily workflow of research in academia and industry.

The Action, ending this month, has given the opportunity to its members to tentatively presenting a set of preliminary requirements to develop further the harmonisation of standards. We hope this will inspire other Actions for the future.

\section{Contacts:}

Domenica D'Elia - Science Communication Manager Susanne Hollmann - Action Chair

Erik Bongcam-Rudloff - Action Vice Chair

Action website: https://www.cost-charme.eu/

\section{Acknowledgements}

The authors thank all the members of the CHARME network (COST Action CA15110) for their contribution.

The CHARME COST Action (CA15110) has been funded by COST - The European Cooperation in Science and Technology, MoU - 042/15.
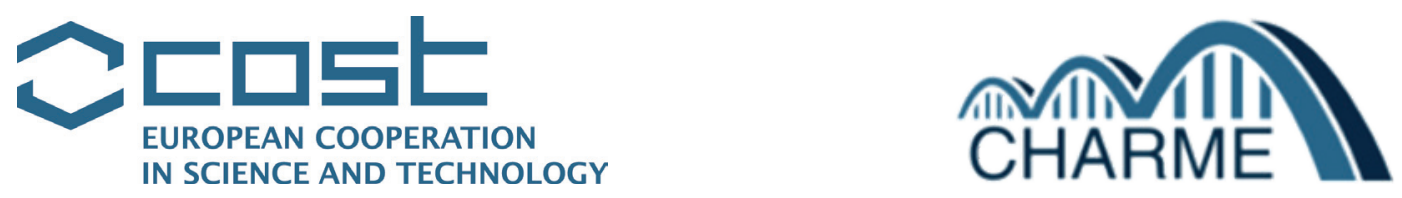